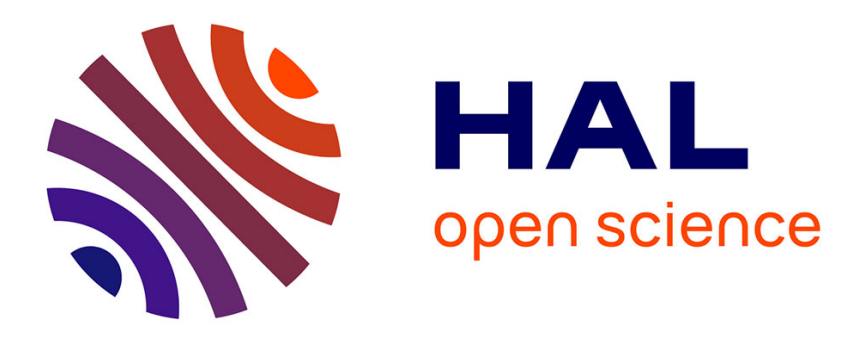

\title{
A global optimization approach for rational sparsity promoting criteria
}

Marc Castella, Jean-Christophe Pesquet

\section{To cite this version:}

Marc Castella, Jean-Christophe Pesquet. A global optimization approach for rational sparsity promoting criteria. EUSIPCO 2017 - 25th European Signal Processing Conference, Aug 2017, Kos Island, Greece. pp.156 - 160, 10.23919/EUSIPCO.2017.8081188 . hal-01666536

\section{HAL Id: hal-01666536 https://hal.science/hal-01666536}

Submitted on 18 Dec 2017

HAL is a multi-disciplinary open access archive for the deposit and dissemination of scientific research documents, whether they are published or not. The documents may come from teaching and research institutions in France or abroad, or from public or private research centers.
L'archive ouverte pluridisciplinaire HAL, est destinée au dépôt et à la diffusion de documents scientifiques de niveau recherche, publiés ou non, émanant des établissements d'enseignement et de recherche français ou étrangers, des laboratoires publics ou privés. 


\section{A Global Optimization Approach for Rational Sparsity Promoting Criteria}

\author{
Marc Castella \\ SAMOVAR, Télécom SudParis, CNRS, \\ Université Paris-Saclay, \\ 9 rue Charles Fourier, 91011 Evry Cedex, France \\ Email: marc.castella@telecom-sudparis.eu
}

\author{
Jean-Christophe Pesquet \\ Center for Visual Computing, CentraleSupelec, \\ Université Paris-Saclay, \\ Grande Voie des Vignes, 92295, Châtenay-Malabry, France \\ Email: jean-christophe@pesquet.eu
}

\begin{abstract}
We consider the problem of recovering an unknown signal observed through a nonlinear model and corrupted with additive noise. More precisely, the nonlinear degradation consists of a convolution followed by a nonlinear rational transform. As a prior information, the original signal is assumed to be sparse. We tackle the problem by minimizing a least-squares fit criterion penalized by a Geman-McClure like potential. In order to find a globally optimal solution to this rational minimization problem, we transform it in a generalized moment problem, for which a hierarchy of semidefinite programming relaxations can be used. To overcome computational limitations on the number of involved variables, the structure of the problem is carefully addressed, yielding a sparse relaxation able to deal with up to several hundreds of optimized variables. Our experiments show the good performance of the proposed approach.
\end{abstract}

\section{INTRODUCTION}

Over the last decade, much attention has been paid to inverse problems involving sparse signals. A popular approach for solving such problems consists in minimizing the sum of a data fidelity term and a regularization term incorporating prior information. When the observation model is linear and the noise has a log-concave likelihood, a convex cost function is obtained. Many efforts have then been dedicated to derive efficient algorithms able to deal with a large number of variables, while ensuring convergence to a global minimizer [1]-[3]. As we will explain now, such a convex formulation may however be limited for two main reasons.

Firstly, for many real acquisition devices, the actual degradation model is not linear and some nonlinear saturation effects often arise. A simplified model then results from a linearization procedure which overlooks these nonlinear behaviors in order to make the associated mathematical problem tractable. For example, standard tools in signal processing such as the Wiener filter are effective mostly in a linear framework. For a long time, attempts have been made in order to deal with more general nonlinear models. For example, one can mention the works undertaken by using Volterra models [4], which may be useful in some application areas [5]. Secondly, convex regularization terms may be limited, especially for capturing the sparse structure of a signal. Potentials related to the $\ell_{1}$ norm are often employed as surrogates to the natural sparsity measure, which is the $\ell_{0}$ pseudo-norm (count of the number of nonzero components in the signal). Although some theoretical works have promoted the use of the $\ell_{1}$ norm [6], its optimality can only been established under some restrictive assumptions. In turn, cost functions involving the $\ell_{0}$ pseudo-norm lead to NP-hard problems for which reaching a global minimum cannot be guaranteed in general [7]-[9]. Smooth approximations of the $\ell_{0}$ pseudo-norm may appear as good alternative solutions [10]-[13]. However, for most of the existing optimization algorithms (e.g. those based on Majorize-Minimize strategies), only convergence to a local minimum can be expected. One can however mention the recent work in [14] where a Geman-McClure like potential was used for deconvolving a sparse signal. Promising results were then obtained.

In this work, we extend the scope of our preliminary work in [14] by proposing a novel approach for restoring sparse signals degraded by a nonlinear model. More precisely, our contributions in this paper are threefold. First, the proposed approach is able to deal with degradation models consisting of a convolution followed by a pointwise transform. The latter appears as a rational fraction involving absolute values and, as an extension of [14], real-valued quantities are here allowed. Such nonlinearly distorted convolution models may also be encountered in blind source separation [15] and neural networks [16]. Our second contribution is to allow the use of a Geman-McClure like regularization with proven global convergence guaranties to a solution of the associated optimization problem. The last contribution of this work is to devise a sparse relaxation in the spirit of [17] to cope with the resulting rational optimization. Since these general rational optimization methods are grounded on building a hierarchy of semidefinite programs (SDP), such a relaxation plays a prominent role in making these approaches applicable to several hundred of variables as it is common in inverse problems.

In Section II, we state our nonlinear model and define the optimized criterion. Section III describes the main steps of our approach. Section IV provides simulation results and Section V concludes this paper. The set of polynomials in the indeterminates given by vector $\mathbf{x}:=\left(x_{1}, \ldots, x_{T}\right)$ is denoted by $\mathbb{R}[\mathbf{x}]$. 


\section{MODEL AND CRITERION}

\section{A. Sparse signal model}

We consider the problem of recovering a set of unknown samples given by the vector $\overline{\mathbf{x}}:=\left(\bar{x}_{1}, \ldots, \bar{x}_{T}\right)^{\top}$. In our context, we only have access to some measurements related to the original signal through a linear transformation (typically, a convolution) and some nonlinear effects. More precisely, the observation model reads

$$
\mathbf{y}=\phi(\mathbf{H} \overline{\mathbf{x}})+\mathbf{n},
$$

where the vector $\mathbf{y}:=\left(y_{1}, \ldots, y_{T}\right)^{\top}$ contains the observation samples, $\mathbf{n}:=\left(n_{1}, \ldots, n_{T}\right)^{\top}$ is a realization of a random independently and identically distributed (i.i.d.) noise vector, $\mathbf{H} \in \mathbb{R}^{T \times T}$ is a given matrix, and $\phi: \mathbb{R}^{T} \rightarrow \mathbb{R}^{T}$ is a nonlinear function. For simplicity, it is assumed that $\phi$ applies componentwise, that is, for every $\mathbf{u}:=\left(u_{1}, \ldots, u_{T}\right)^{\top}$, the $k$ th component of $\phi(\mathbf{u})$ is given by $[\phi(\mathbf{u})]_{k}=\phi\left(u_{k}\right)$. Here, $\phi$ models a rational saturation effect, which is parametrized by $\delta_{n}>0$, and which is given by:

$$
(\forall u \in \mathbb{R}) \quad \phi(u)=\frac{u}{\delta_{n}+|u|} .
$$

The model (1) appears in the case when the samples stem from a signal given by $y_{t}=\phi\left(h_{t} \star \bar{x}_{t}\right)+n_{t}$ for all $t \in\{1, \ldots, T\}$. In the latter equation, $\star$ denotes the convolution by the filter with impulse response $\left(h_{t}\right)_{t}$. We assume that the convolution filter has a finite impulse response (FIR) given by the vector $\left(h_{1}, \ldots, h_{L}\right)^{\top}$. Under suitable vanishing boundary conditions, the matrix $\mathbf{H}$ is thus Toeplitz banded as shown below:

$$
\mathbf{H}=\left[\begin{array}{cccccc}
h_{1} & 0 & \ldots & \ldots & \ldots & 0 \\
\vdots & \ddots & \ddots & & & \vdots \\
h_{L} & & & \ddots & & \vdots \\
0 & \ddots & & & \ddots & \vdots \\
\vdots & \ddots & \ddots & & \ddots & 0 \\
0 & \ldots & 0 & h_{L} & \ldots & h_{1}
\end{array}\right] .
$$

One of the main novelty of this work is to exploit the specific structure of matrix $\mathbf{H}$ in order to reduce the computational cost of the subsequently proposed global optimization method. Finally, the signal $\left(x_{t}\right)_{t \in \mathbb{Z}}$ is assumed to be sparse, that is we simply assume that $x_{t} \neq 0$ only for a few indices $t$.

\section{B. Criterion for recovery}

Following a classical approach for estimating $\overline{\mathbf{x}}$, we minimize a penalized criterion having the following form:

$$
\left(\forall \mathbf{x} \in \mathbb{R}^{T}\right) \quad \mathcal{J}(\mathbf{x})=\|\mathbf{y}-\boldsymbol{\phi}(\mathbf{H} \mathbf{x})\|^{2}+\lambda \sum_{t=1}^{T} \psi_{\delta}\left(x_{t}\right),
$$

where $\lambda$ and $\delta$ are positive regularization and smoothing parameters, and $\psi_{\delta}$ is a Geman-McClure like potential similar to the one used in [14]:

$$
(\forall \xi \in \mathbb{R}) \quad \psi_{\delta}(\xi)=\frac{|\xi|}{\delta+|\xi|} .
$$

The minimization is performed over a compact feasible set denoted by $\mathbf{K}$ and the optimization problem consists in finding

$$
\mathcal{J}^{\star}:=\inf _{\mathbf{x} \in \mathbf{K}} \mathcal{J}(\mathbf{x}) .
$$

\section{RATIONAL MINIMIZATION}

To solve $\mathcal{J}^{\star}$, we use a methodology similar to [14]. The differences and novelties are described in the next two subsections: first, we can deal with real-valued quantities, and second, we exploit the problem structure.

\section{A. Optimization set and absolute values}

We here specify the feasible set $\mathbf{K}$. From its description, it becomes possible to cope with the absolute values in (2) and (3). First, the optimization set $\mathbf{K}$ mentioned above is described by polynomial inequalities as follows:

$$
\mathbf{K}=\left\{\mathbf{x} \in \mathbb{R}^{T} \mid g_{i}(\mathbf{x}) \geq 0, i=1, \ldots, I\right\} .
$$

Technically, it is required that the above representation of $\mathbf{K}$ provides an algebraic certificate of compactness [18]. In our practical situation, this is easily satisfied when upper and lower bounds on the variables $\left(x_{t}\right)_{1<t<T}$ are available: in this case indeed, $\mathbf{K} \subset[-B, B]^{T}$ and hence the polynomials corresponding to the inequalities $g_{t}\left(x_{t}\right)=-\left(x_{t}+B\right)\left(x_{t}-B\right) \geq 0$ can be included in the polynomials in (5). Details on these technical conditions are out of the scope of this paper and can be found in $[18,19]$.

Furthermore, we can use the above specification of the feasible set $\mathbf{K}$ to handle absolute values. First, by introducing a polynomial and its opposite in (5), it is possible to introduce polynomial equality constraints in $\mathbf{K}$. Then, absolute values can be considered as follows: for each term $|\tilde{u}(\mathbf{x})|$ appearing, where $\tilde{u}$ is a polynomial, add an additional variable $u$ and impose the constraints $u \geq 0, u^{2}=\tilde{u}(\mathbf{x})^{2}$. The methodology described in Section III-C of this paper can then be applied with the extended set of variables $(\mathbf{x}, u)$.

\section{B. Structure of the problem}

Developing the square norm in (3) and substituting all terms, the criterion $\mathcal{J}$ appears as a sum of rational functions, possibly involving absolute values. The absolute values can be eliminated using the trick of the previous paragraph: for clarity, and with no loss of generality, we describe the method when all quantities are nonnegative and hence no absolute values appear.

We take advantage of the Toeplitz banded structure of $\mathbf{H}$, and introduce a sparse ${ }^{1}$ relaxation of $\mathcal{J}^{\star}$. This technique was introduced in a different context [17]. Define for each $t \in\{1, \ldots, T\}$ the set $I_{t}=\{\min \{1, t-L+1\}, \ldots, t\}$ of

\footnotetext{
${ }^{1}$ The notion of sparsity here concerns the SDP relaxation and should not be confused with the sparsity assumed for the original samples of vector $\overline{\mathbf{x}}$.
} 
column indices of $\mathbf{H}$ for which row $t$ has nonzero elements. Developing the square norm in (3), we write $\mathcal{J}(\mathbf{x})$ as follows:

$$
\mathcal{J}(\mathbf{x})=\sum_{t=1}^{T} \underbrace{\left[y_{t}-\phi\left(\sum_{i=1}^{L} h_{i} x_{t-i+1}\right)\right]^{2}}_{\frac{p_{I_{t}}(\mathbf{x})}{q_{I_{t}}(\mathbf{x})}}+\underbrace{\lambda \psi_{\delta}\left(x_{t}\right)}_{\frac{p\left(x_{t}\right)}{q\left(x_{t}\right)}},
$$

with the convention that $x_{t}=0$ for $t \notin\{1, \ldots, T\}$. In other words, $p_{I_{t}}, q_{I_{t}}$ are polynomials that depend on the variables $\left(x_{k}\right)_{k \in I_{t}}$ only and $p, q$ are univariate polynomials that depend on $x_{t}$ only. Define the following sets:

$$
(\forall t \in\{1, \ldots, T\}) \quad J_{t}=I_{t} \text { and } J_{t+T}=\{t\} .
$$

The sets $\left(J_{t}\right)_{1 \leq t \leq 2 T}$ satisfy $\bigcup_{t=1}^{2 T} J_{t}=\{1, \ldots, T\}$ and the socalled Running Intersection Property (RIP), that is for every $t \in\{2, \ldots, 2 T\}$ :

$$
J_{t} \bigcap\left(\bigcup_{k=1}^{t-1} J_{k}\right) \subseteq J_{j} \text { for some } j \leq t-1 .
$$

Consequently, the methodology in [17] is applicable.

\section{Sparse SDP relaxation}

Similarly to [14], $\mathcal{J}^{\star}$ can be relaxed to a finite dimensional SDP, where each optimization variable corresponds to a monomial in $\left(x_{1}, \ldots, x_{T}\right)$. We briefly sketch the main steps. First, it can be proved that the original problem is equivalent to an optimization problem over several finite Borel measures, which is called a generalized moment problem. Each measure is then represented by a truncated moment sequence $\mathbf{y}$. For any such moment sequence, we define the following linear functional, which replaces any monomial in a given polynomial $f \in \mathbb{R}[\mathbf{x}]$ by the corresponding moment value in $\mathbf{y}$ :

$$
\begin{aligned}
L_{\mathbf{y}}: \quad \mathbb{R}[\mathbf{x}] & \rightarrow \mathbb{R} \\
& f=\sum f_{\boldsymbol{\alpha}} \mathbf{x}^{\boldsymbol{\alpha}} \mapsto \sum f_{\boldsymbol{\alpha}} y_{\boldsymbol{\alpha}} .
\end{aligned}
$$

In the relaxation below, $L_{\mathbf{y}}(f)$ will correspond to the value taken by a polynomial $f$ for a given measure represented by the moment sequence $\mathbf{y}$. For any order $k \in \mathbb{N}$ and for multiindices $\boldsymbol{\alpha}, \boldsymbol{\beta}$ of order $|\boldsymbol{\alpha}|:=\alpha_{1}+\cdots+\alpha_{n} \leq k$ and $|\boldsymbol{\beta}| \leq k$, the moment matrix of $\mathbf{y}$ is defined by

$$
\left[M_{k}(\mathbf{y})\right]_{\boldsymbol{\alpha}, \boldsymbol{\beta}}:=y_{\boldsymbol{\alpha}+\boldsymbol{\beta}},
$$

and for a given polynomial $g \in \mathbb{R}[\mathbf{x}]$, the localizing matrix associated with $g$ and $\mathbf{y}$ is

$$
\left[M_{k}(g \mathbf{y})\right]_{\boldsymbol{\alpha}, \boldsymbol{\beta}}:=\sum_{\boldsymbol{\gamma}} g_{\boldsymbol{\gamma}} y_{\boldsymbol{\gamma}+\boldsymbol{\alpha}+\boldsymbol{\beta}} .
$$

The two above matrices, will make it possible in the relaxation to introduce the conditions such as $M_{k}(\mathbf{y}) \succeq 0$ and $M_{k}(g \mathbf{y}) \succeq 0$, which are necessary for $\mathbf{y}$ to represent a measure concentrated on the set where $g$ is nonnegative. Finally, the $k^{\text {th }}$ order sparse SDP relaxation consists in the minimization problem

$$
\mathcal{P}_{k}^{\star \mathrm{s}}:=\inf \sum_{t=1}^{T} L_{\mathbf{z}_{t}}\left(p_{I_{t}}\right)+L_{\mathbf{y}_{t}}(p)
$$

subject to a number of semidefinite positivity constraints and linear equality constraints (see [17] for mathematical details). Note in particular that, in order to deal with the fractions in $\mathcal{J}(\mathbf{x})$, the constraints $L_{\mathbf{z}_{t}}\left(q_{I_{t}}\right)=L_{\mathbf{y}_{t}}(q)=1$ on the denominator values should appear.

With increasing values of $k$, this yields a hierarchy of SDP relaxations for which it can be proved [17] that $\mathcal{P}_{k}^{\star \mathrm{s}} \uparrow \mathcal{J}^{\star}$ as $k \rightarrow \infty$. Moreover, from $\mathcal{P}_{k}^{\star \mathrm{s}}$, a point $\mathbf{x}_{k}^{\star \mathrm{s}}$ can be extracted, which is guaranteed to be optimal under certain rank conditions and possibly only approximate otherwise.

The above relaxation is similar to the one in [14]. Based on the split form of $\mathcal{J}(\mathbf{x})$, the moment sequences $\mathbf{y}_{t}$ correspond to monomials involving the variables $\left(x_{k}\right)_{k \in J_{t}}$ which appear in the respective terms $\frac{p_{I_{t}}(\mathbf{x})}{q_{I_{t}}(\mathbf{x})}$. Similarly, the moment sequences $\mathbf{z}_{t}$ correspond to monomials involving $x_{t}$ only, in accordance to the variable appearing in the terms $\frac{p\left(x_{t}\right)}{q\left(x_{t}\right)}$. Of course, the same subset of variables may appear in different moment sequences $\mathbf{y}_{t}$ or $\mathbf{z}_{t}$ : therefore compatibility conditions are required, corresponding to equality of moments. This is made possible by the RIP condition and it justifies that linear equality constraints are necessary in $\mathcal{P}^{\star s}$.

Importantly, and in contrast with [14], no moment sequence involves all variables $\left(x_{t}\right)_{1 \leq t \leq T}$. As a consequence, the semidefinite positivity constraints in $\mathcal{P}_{k}^{\star s}$ only involve small subsets of the whole set of variables $\left(x_{t}\right)_{1<t<T}$. This constitutes the great benefit of this sparse relaxation, allowing us to deal with a much higher number of variables. A striking example illustrating this fact is when the criterion is separable and the terms in $\mathcal{J}(\mathbf{x})$ all involve distinct variables.

\section{Simulations}

\section{A. Experimental setup}

We have generated samples $\overline{\mathbf{x}}$ from a sparse signal. The number of samples in $\overline{\mathbf{x}}$ has been set to $T=200, T=100$, $T=50$ and $T=20$. We have imposed that exactly $10 \%$ of the samples are nonzero, yielding respectively $20,10,5$ and 2 nonzero components in $\overline{\mathbf{x}}$. The amplitudes of the nonzero components have been drawn according to a uniform distribution on $[-1 ;-2 / 3] \cup[2 / 3 ; 1]$. Then, this unknown impulsive signal has been corrupted according to Model (1) with the nonlinearity given by (2). We have set $\delta_{n}=0.3$ and the noise $\mathbf{n}$ has been drawn according to an i.i.d. zero-mean Gaussian distribution with standard deviation $\sigma=0.15$. Finally, the matrix $\mathbf{H}$ has been set Toeplitz banded corresponding to FIR filters of length 3. Several sets of 100 Monte-Carlo realizations of such data have been generated, both with fixed FIR filter with impulse responses given by $\mathbf{h}^{(a)}=[0.1,0.8,0.1]$ and by $\mathbf{h}^{(b)}=[0.3871,-0.1887,0.4242]$ and with impulse responses drawn randomly for each Monte-Carlo realization. We have set empirically $\lambda=0.15$ for the regularization parameter and $\delta=0.01$ in the penalty function (4). To obtain an estimate $\mathbf{x}_{3}^{\star \mathrm{s}}$, 


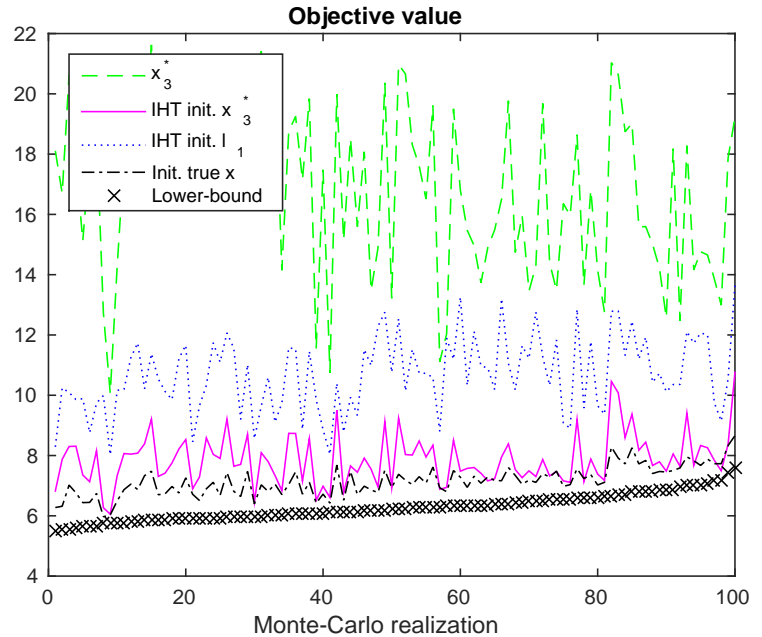

Fig. 1. For all realizations, lower bound and objective value attained by our method; objective value attained by IHT with different initializations (filter with random coefficient, $T=200)$.

we have solved the SDP relaxation $\mathcal{P}_{3}^{\star \mathrm{s}}$ of order $k=3$ from Section III-C. We are not aware of any other method able to find the global minimum of (3) and for comparison, we have used a linearized model: for reconstruction purposes, using data generated according to (1), we have linearized around zero the nonlinearity in (2) and have used the well-known $\ell_{1}$ penalization.

Finally, we have also implemented a proximal gradient algorithm based on Iterative Hard Thresholding (IHT) [8] extended to the nonlinear model. The latter algorithm however only certifies convergence to a local minimum of the criterion. Due to non convexity, the local minima are likely to be different from the global minimum.

\section{B. Performance of the proposed relaxation}

Note first that, for the order of relaxation $k=3$, we have been able to consider up to $T=200$ samples with the sparse relaxation used here. This is a significant improvement in comparison with the results in [14], where the relaxation was able to deal with only a few tens of variables with an order of relaxation not greater than 3 .

We have plotted on Figure 1 the lower bound provided by the relaxation $\mathcal{P}_{3}^{\star \mathrm{s}}$ and the objective value $\mathcal{J}\left(\mathbf{x}_{3}^{\star \mathrm{s}}\right)$ attained when extracting an optimal point from the same order relaxation. We clearly see that $\mathcal{P}_{3}^{\star \mathrm{s}} \leq \mathcal{J}\left(\mathbf{x}_{3}^{\star \mathrm{s}}\right)$, which is in accordance with the theory. However, equality does not perfectly hold. This illustrates that the choice $k=3$ of the relaxation order is probably too small for the considered problem. As a consequence, we propose in next section to combine our method with a local IHT optimization method.

\section{Existence of local minima and results of the proposed} global approach

The IHT algorithm has been initialized with $\mathbf{x}_{3}^{\star s}$, the result from the linearized model and $\ell_{1}$ penalization, $\mathbf{y}$, an all- zero vector and the true $\overline{\mathbf{x}}$ (the latter initialization would be intractable in real applications). The average values over all Monte-Carlo realizations are provided in Table I for $T=200$. Some detailed values, corresponding to randomly drawn filter coefficients, are plotted in Figure 1, also for $T=200$.

The final objective values after convergence of the IHT optimization clearly depend on the initialization, which advocates in favor of the existence of several local optima and emphasizes the importance of considering the problem of global optimization. In average, the lowest objective value is obtained by a local optimization initialized either at $\mathbf{x}_{3}^{\star s}$ or at the true $\overline{\mathbf{x}}$. More importantly, as shown in Table II, the IHT algorithm seems unable to reach the global minimum with any of the initializations easily available $\left(\ell_{1}, \mathbf{y}\right.$, all-zero vector). This shows clearly that the proposed relaxation is very useful in providing an improved initialization point for a local optimization algorithm.

TABLE I

FINAL VALUES OF THE OBJECTIVE $\mathcal{J}(\mathbf{x})$ FOR THE NONLINEAR LOCAL OPTIMIZATION METHODS.

\begin{tabular}{|l||c|c|c|}
\hline \multirow{2}{*}{ Opt. method } & \multicolumn{3}{c|}{ Filter param. } \\
\cline { 2 - 4 } & $\mathbf{h}^{(a)}$ & $\mathbf{h}^{(b)}$ & random \\
\hline \hline $\mathbf{X}_{3}^{\star \mathrm{S}}$ & 12.181 & 20.852 & 16.776 \\
linearized $\ell_{1}$ & 22.032 & 19.719 & 20.848 \\
\hline IHT, init. $\mathbf{x}_{3}^{\star \mathrm{S}}$ & 7.2900 & 8.9858 & 7.8665 \\
IHT, init. $\ell_{1}$ & 10.157 & 11.186 & 10.690 \\
IHT, init. $\mathbf{y}$ & 10.167 & 12.505 & 13.144 \\
IHT, init. zero & 12.231 & 15.417 & 13.392 \\
IHT, init. $\mathbf{\mathbf { x }}$ & 7.1992 & 7.1578 & 7.1489 \\
\hline
\end{tabular}

TABLE II

OUt OF 100 MONTE-CARLO REALIZATIONS, NUMBER OF TIMES EACH INITIALIZATION OF IHT PROVIDES THE SMALLEST OBJECTIVE VALUE $\left(\right.$ FILTER $\left.^{(a)}, T=200\right)$.

\begin{tabular}{|c|c|c|c|c|c|}
\hline \multirow{2}{*}{$\begin{array}{l}\text { Num. } \\
\text { samples }\end{array}$} & \multicolumn{5}{|c|}{ Initialization } \\
\hline & $\mathbf{x}_{3}^{\star \mathrm{S}}$ & $\ell_{1}$ & $\mathbf{y}$ & zero & $\overline{\mathbf{x}}$ \\
\hline 20 & 56 & 0 & 1 & 18 & $\overline{440}$ \\
\hline 50 & 38 & 0 & 0 & 0 & 62 \\
\hline 100 & 36 & 0 & 0 & 0 & 64 \\
\hline 200 & 22 & 0 & 0 & 0 & 78 \\
\hline
\end{tabular}

\section{Results on signal recovery}

Finally, we illustrate the ability of our method to estimate the sought signal. A typical example of the true $\bar{x}$, of the observation vector $\mathrm{y}$ and of the reconstructed signal is given on Figure 2. The estimation error on $\overline{\mathbf{x}}$ has been quantified by the mean square error $\frac{1}{T}\|\hat{\mathbf{x}}-\overline{\mathbf{x}}\|^{2}$ for a given estimate $\hat{\mathbf{x}}$. The average error and objective values are gathered in Table III and show poor results are obtained with a linearized model. The best results have been obtained by IHT initialized by $\mathbf{x}_{3}^{\star s}$. The result of IHT initialized by the true $\overline{\mathbf{x}}$ is given for information.

\section{CONCLUSION}

We have considered sparse signals observed through a nonlinear observation model with additive noise. The class of nonlinear models that can theoretically be tackled with our methodology is quite vast, as it includes any rational function, possibly involving absolute values. 

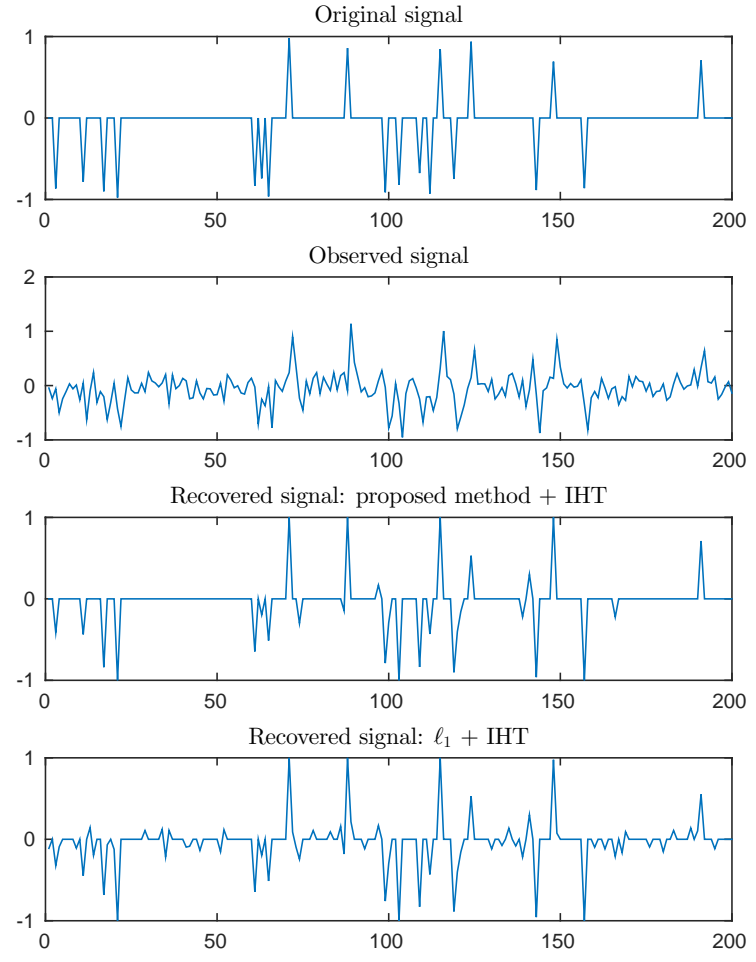

Fig. 2. Typical original signal $\overline{\mathbf{x}}$, observations $\mathbf{y}$ and recovered signal. The results presented have been obtained with IHT initialized either by our method or by a linearized model and $\ell_{1}$ penalty.

TABLE III

FiNAL AVERAGE MSE FOR THE NONLINEAR LOCAL OPTIMIZATION METHODS.

\begin{tabular}{|l||c|c|c|}
\hline \multirow{2}{*}{ Opt. method } & \multicolumn{3}{c|}{ Filter param. } \\
\cline { 2 - 4 } & $\mathbf{h}^{(a)}$ & $\mathbf{h}^{(b)}$ & random \\
\hline \hline $\mathbf{x}_{3}^{\star \mathrm{s}}$ & $8.40 \mathrm{e}-3$ & $1.57 \mathrm{e}-2$ & $1.29 \mathrm{e}-2$ \\
linearized $\ell_{1}$ & $3.88 \mathrm{e}-2$ & $3.15 \mathrm{e}-2$ & $3.38 \mathrm{e}-2$ \\
\hline IHT, init. $\mathbf{x}_{3}^{\star \mathrm{s}}$ & $8.95 \mathrm{e}-3$ & $1.45 \mathrm{e}-2$ & $1.22 \mathrm{e}-2$ \\
IHT, init. $\ell_{1}$ & $1.30 \mathrm{e}-2$ & $2.14 \mathrm{e}-2$ & $1.95 \mathrm{e}-2$ \\
IHT, init. $\mathbf{y}$ & $2.60 \mathrm{e}-2$ & $2.34 \mathrm{e}-2$ & $3.96 \mathrm{e}-2$ \\
IHT, init. zero & $5.20 \mathrm{e}-2$ & $6.60 \mathrm{e}-2$ & $5.57 \mathrm{e}-2$ \\
IHT, init. $\overline{\mathbf{x}}$ & $5.18 \mathrm{e}-3$ & $4.08 \mathrm{e}-3$ & $4.49 \mathrm{e}-3$ \\
\hline
\end{tabular}

The starting point of our reconstruction approach involves the minimization of a criterion which is the sum of a fidelity term and a penalization. The fidelity has been chosen as a square norm and the penalization as a Geman-McClure potential approaching the $\ell_{0}$ norm. It has been known for long that such problems are particularly hard to solve. However, with the adopted formulation and approximation, the optimization problem is rational, which opens up the possibility to use recent methodologies with theoretical global convergence properties.

The desirable convergence properties of the latter methodologies come at the cost of solving a high dimensional SDP. With state of the art SDP solvers, this limits the approach to small size problems. To overcome these computational limitations, we have exploited a particular model structure. In so doing, we have been able to deal with up to 200 variables. More specifically, the proposed methodology seems promising in order to provide an initialization point to a local algorithm such as IHT. Finally, the results in terms of reconstruction seem promising.

\section{REFERENCES}

[1] P. L. Combettes and J.-C. Pesquet, "Proximal splitting methods in signal processing," in Fixed-Point Algorithms for Inverse Problems in Science and Engineering, H. H. Bauschke, R. Burachik, P. L. Combettes, V. Elser, D. R. Luke, and H. Wolkowicz, Eds. New York: SpringerVerlag, 2010, pp. 185-212.

[2] N. Komodakis and J.-C. Pesquet, "Playing with duality: an overview of recent primal-dual approaches for solving large-scale optimization problems," IEEE Signal Process. Mag., vol. 32, pp. 31-54, Nov. 2015.

[3] S. Boyd, N. Parikh, E. Chu, B. Peleato, and J. Eckstein, "Distributed optimization and statistical learning via the alternating direction method of multipliers," Found. Trends Machine Learn., vol. 8, no. 1, pp. 1-122, 2011.

[4] M. Shetzen, The Volterra and Wiener Theories of Nonlinear Systems. New York: Wiley and sons, 1980.

[5] N. Dobigeon, J.-Y. Tourneret, C. Richard, J. C. M. Bermudez, S. McLaughlin, and A. O. Hero, "Nonlinear unmixing of hyperspectral images: models and algorithms," IEEE Signal Process. Mag., vol. 31, no. 1, pp. 82-94, Jan. 2014.

[6] E. J. Candès and M. B. Wakin, "An introduction to compressive sampling," IEEE Signal Process. Mag., vol. 25, no. 2, pp. 21-30, Mar. 2008.

[7] M. Nikolova, "Description of the minimizers of least squares regularized with $\ell_{0}$ norm. Uniqueness of the global minimizer," SIAM J. Imaging Sci., vol. 6, no. 2, pp. 904-937, 2013.

[8] T. Blumensath and M. E. Davies, "Iterative thresholding for sparse approximations," J. Fourier Anal. Appl., vol. 14, no. 5-6, pp. 629-654, 2008.

[9] A. Patrascu and I. Necoara, "Random coordinate descent methods for $\ell_{0}$ regularized convex optimization," IEEE Trans. Automat. Contr., vol. 60, no. 7, pp. 1811-1824, Jul. 2015.

[10] S. Geman and D. McClure, "Bayesian image analysis: An application to single photon emission tomography," in Proc. Statist. Comput. Section Amer. Statist. Association, 1985, pp. 12-18.

[11] E. Chouzenoux, A. Jezierska, J.-C. Pesquet, and H. Talbot, "A majorizeminimize subspace approach for $\ell_{2}-\ell_{0}$ image regularization," SIAM $J$. Imaging Sci., vol. 6, no. 1, pp. 563-591, 2013.

[12] A. Florescu, E. Chouzenoux, J.-C. Pesquet, P. Ciuciu, and S. Ciochina, "A majorize-minimize memory gradient method for complex-valued inverse problem," Signal Process., vol. 103, pp. 285-295, Oct. 2014, special issue on Image Restoration and Enhancement: Recent Advances and Applications.

[13] E. Soubies, L. Blanc-Féraud, and G. Aubert, "A continuous exact $\ell_{0}$ penalty (CEL0) for least squares regularized problem," SIAM J. Imaging Sci., vol. 8, no. 3, pp. 1607-1639, 2015.

[14] M. Castella and J.-C. Pesquet, "Optimization of a Geman-McClure like criterion for sparse signal deconvolution," in Proc. IEEE Int. Workshop on Computational Advances in Multi-Sensor Adaptive Processing (CAMSAP), Cancun, Mexico, Dec. 2015, pp. 317-320.

[15] Y. Deville and L. T. Duarte, "An overview of blind source separation methods for linear-quadratic and post-nonlinear mixtures," in Proc. of the 12th Int. Conf. LVA/ICA, ser. LNCS, vol. 9237. Liberec, Czech Republic: Springer, 2015, pp. 155-167.

[16] D. J. C. MacKay, Information Theory, Inference, and Learning Algorithms. Cambridge University Press, 2003.

[17] F. Bugarin, D. Henrion, and J.-B. Lasserre, "Minimizing the sum of many rational functions," Mathematical Programming Computations, vol. 8, no. 1, pp. 83-111, 2015.

[18] J.-B. Lasserre, Moments, Positive Polynomials and Their Applications, ser. Optimization Series. Imperial College Press, 2010, vol. 1.

[19] M. Laurent, "Sum of squares, moment matrices and optimization over polynomials," in Emerging Applications of Algebraic Geometry, ser. IMA Volumes in Mathematics and its Applications, M. Putinar and S. Sullivant, Eds. Springer, 2009, vol. 149, pp. 157-270. 